\title{
Toward the Optimal Lead System and Optimal Criteria for Exercise Electrocardiography
}

\author{
MAARTEN L. SIMOONS, .MD \\ PIERRE BLOCK, MD
}

Rotterdam, The Netherlands

Brussels, Belgium
From the Thoraxcenter, Erasmus University, Rotterdam, The Netherlands, and Unit for Cardiovascular Research, University Hospital of Jette, VUB Brussels, Belgium. Manuscript received March 5, 1981, accepted March 13, 1981.

Address for reprints: Maarten L. Simoons, MD, 77 Thoraxcenter, Erasmus University, PO Box 1738, Rotterdam, The Netherlands.
To define the optimal lead system for exercise electrocardiography, data of the whole body surface potentlal distribution were analyzed in 25 normal subjects and in 25 patients with coronary artery disease at rest and during exerclse. All patlents had a normal electrocardiogram at rest. The sensitivity of the standard chest leads was 60 percent; it improved to 84 percent with the body surface map whereas both methods had a 100 percent specificity. On the basis of these data, and reports from other centers, $\boldsymbol{H}$ is concluded that a single bipolar lead from the right subclavian area to lead $V_{5}$ is adequate in those laboratories that are restricted to testing subjects with a normal electrocardiogram at rest. In patients with a previous infarction or other abnormalities in the electrocardiogram at rest three (pseudo) orthogonal leads or several standard leads are necessary.

Recommendations for optimal measurements from the exercise electrocardiogram are based on quantitative computer analysis of selected leads in larger groups of patients. Best results were obtained with a combination of S-T amplitude, S-T slope and heart rate. The improvement in sensitivity from 50 percent with visual analysis to 85 percent with computer was similar to that obtained with body surface mapping. Changes of the P wave and QRS complex during exercise appeared to be of little dlagnostic value. The pathophysiologic mechanisms that contribute to the changes of the electrocardiogram during exercise are discussed.

The first recordings of the electrocardiogram after exercise were made in 1908 by Einthoven, ${ }^{1}$ the founder of electrocardiography. The clinical application of postexercise electrocardiography was introduced almost 30 years later and reviewed by Scherf and Schaffer. ${ }^{2}$ In recent years there has been a steady development toward the recording of multiple leads during the entire stress test as well as the quantitative analysis of electrocardiographic changes during and after exercise. However, opinions still differ on basic questions such as selection of leads and interpretation of changes in the electrocardiogram during exercise. The answers to these questions may be obtained from quantitative analysis of the whole body surface potential distribution during exercise.

In this study recommendations are made concerning the choice of lead systems in various clinical settings and the choice of electrocardiographic measurements to be taken during exercise. These recommendations are based on a combination of body surface data in selected patients and quantitative data from selected leads in larger groups of patients. This discussion is restricted to the electrocardiogram. However, other data obtained during an exercise test are of equal value, such as exercise capacity, heart rate and blood pressure responses and the occurrence of chest pain or dyspnea. 


\section{Body Surface Potential Distribution at Rest and During Exercise}

Body surface maps were recorded with 120 electrodes taped to the chest wall. ${ }^{3}$ The recording system has been described in detail. ${ }^{4}$ Eight seconds of data were recorded from all leads simultaneously with adequate frequency response and with a resolution of $20 \mu \mathrm{V}$ in digital format. After visual inspection of the tracings, beats with similar waveforms were averaged in order to obtain single representative complexes with a low noise level at rest and during exercise. The onset and end of the QRS interval were determined with a computer program and checked by the operator. ${ }^{5}$ Exercise was performed on a bicycle ergometer with stepwise increments in work load of 25 watts every 5 minutes. ${ }^{3}$ Data were recorded with the patient at rest seated on the bicycle ergometer, at peak exercise and in the 1st minute of the recovery period.

Body surface maps in normal subjects: Data were analyzed from 25 normal men, aged 28 to 59 years. These subjects had no history of cardiovascular disease and were selected on the basis of normal findings on 12 lead electrocardiography, chest X-ray study, exercise testing and postexercise thallium scintigraphy. ${ }^{6}$ Coronary arteriography was not performed in these subjects. The peak heart rate during exercise averaged 150 beats/min (range 135 to 175 ).

Ventricular depolarization at rest could be characterized by a maximum in the sternal region that shifted gradually toward the left lateral area and by a minimum at the back or the right shoulder that moved toward the sternum. In most patients a second maximum developed at the end of depolarization in the right subclavian area (Fig. 1). Repolarization was characterized by a maximum at the mid sternum or at the left sternal border and a minimum that moved from the back toward the anterior part of the right shoulder. These observations were similar to those reported by Taccardi ${ }^{7}$ and other investigators. 8,9

During exercise the depolarization pattern was similar to that at rest (Fig. 2). In 15 of 25 subjects the amplitudes of the maximums and minimums increased $0.1 \mathrm{mV}$ or more, up to $0.77 \mathrm{mV}$, whereas the amplitudes decreased $0.1 \mathrm{mV}$ or more in six subjects (Fig. 3). A reduction of the maximal QRS magnitude was described recently in 20 young subjects. ${ }^{10}$ The early repolarization pattern during exercise differed from the pattern at rest. In the first 10 to $20 \mathrm{~ms}$ the potential distribution was similar to the late depolarization patterns. The precordial minimum remained present during 40 to $100 \mathrm{~ms}$ after the end of the QRS complex. The amplitude of this minimum was low: less than $90 \mu \mathrm{V}$ in all subjects at 60 ms after the end of the QRS complex.

The late repolarization pattern was again similar to the pattern at rest, although the voltages reached the highest values earlier. In 12 subjects the repolarization voltages had the same magnitudes at rest and during exercise whereas in 8 subjects the voltages during exercise were smaller and in 5 they were greater. Mirvis et al. ${ }^{11}$ described precordial maps during strenuous

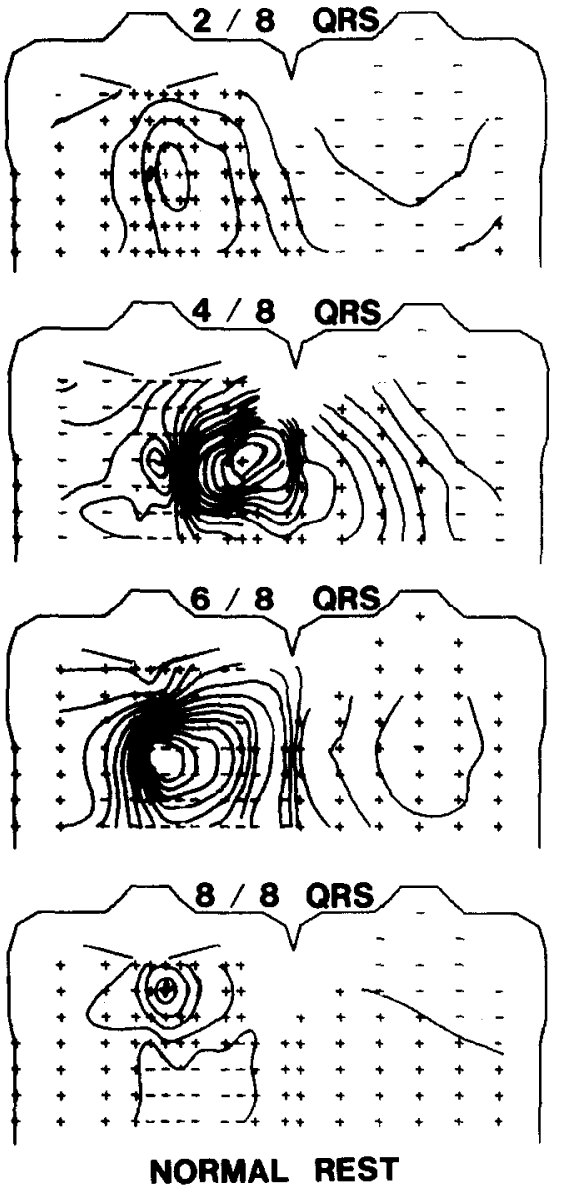

FICURE 1. Body surface maps during depolarization in a normal subject at rest. In each dlagram the left part represents the anterior chest wall and the right represents the back. Two lines that represent the clavicles have been drawn in each dlagram as well as in the other surface maps. The maps correspond to four time normalized instants during depolarization at $2 / 8,4 / 8,6 / 8$ and $8 / 8$ of QRS duration. Note a maximum in the sternal region during early depolarization (2/8), that shitts toward the left $(4 / 8)$ and the back $(6 / 8)$. At the end of depolarization two maximums are present, at the back and at the upper part of the sternum $(8 / 8)$. The minimum shifts from the back $(2 / 8)$ through the right side of the chest $(4 / 8)$ toward the left precordial region $(6 / 8$ and $8 / 8)$.

exercise in 15 normal volunteers. These authors observed similar negative precordial potentials during early repolarization as previously described.

Body surface maps in patients with coronary artery disease: Body surface maps were made in 25 men with coronary artery disease between age 40 and 55 years. All had a normal QRS complex and S-T segment at rest, and no history of myocardial infarction. Selective coronary arteriography showed 50 percent or greater luminal narrowing in one or more major coronary arteries. These patients underwent the same exercise test as the normal subjects. They recorded a peak heart rate of 135 beats/min (range 110 to 60 ).

The depolarization maps were similar to the normal maps in 23 patients. Two patients had abnormal negative voltages at the right anterior chest wall during 
early depolarization. Repolarization at rest was abnormal in five patients, in spite of a normal 12 lead electrocardiogram. These patients had a negative precordial potential that exceeded $-50 \mu \mathrm{V} 60 \mathrm{~ms}$ after the QRS complex. During exercise the depolarization potentials increased by $0.1 \mathrm{mV}$ or more in 15 of 25 patients (Fig. 3) and decreased by this amount in 5 patients. Thus, no systematic difference was observed between the direction or the magnitude of the QRS changes in the normal subjects and in the patients. In six patients the late maximum at the right shoulder or at the right sternal border was increased.

The greatest deviation from the normal pattern was observed in the early repolarization during exercise (Fig. 4). In all patients a prolonged negative area developed at the precordium. The precise location of the greatest negative values varied (Fig. 5). ${ }^{11,12}$ However, no relation could be observed between the repolarization pattern and the location of defects in the thallium scintigrams or the location of coronary arterial obstruction; this finding confirms data in earlier reports from our laboratory ${ }^{13}$ and others. ${ }^{14}$

\section{Diagnostic Value of Exercise Surface Maps}

In some patients with coronary artery disease, the surface maps showed abnormal repolarization patterns that were not present in the standard 12 lead electrocardiogram. A horizontal or downsloping S-T segment depression in the standard chest leads was present in only 15 of the 25 patients. Nevertheless 21 patients had abnormal negative precordial potentials greater than $90 \mu \mathrm{V} 60 \mathrm{~ms}$ after the end of QRS complex, whereas such potentials were not present in a single normal subject. The other four patients had a negative precordial area between 50 and $90 \mu \mathrm{V}$. Such negative values were also present in three normal subjects (Fig. 5 and 6). The results of this analysis are presented in Table I.

Similar results were presented by Fox et al. ${ }^{12}$ in a series of 100 patients with 16 lead precordial maps. In their study the sensitivity was 96 percent with the maps and 80 percent with the 12 lead system whereas the specificity of both methods was the same. The body surface data were obtained at rest, at peak exercise and in the 1st minute of the recovery period. Further anal-
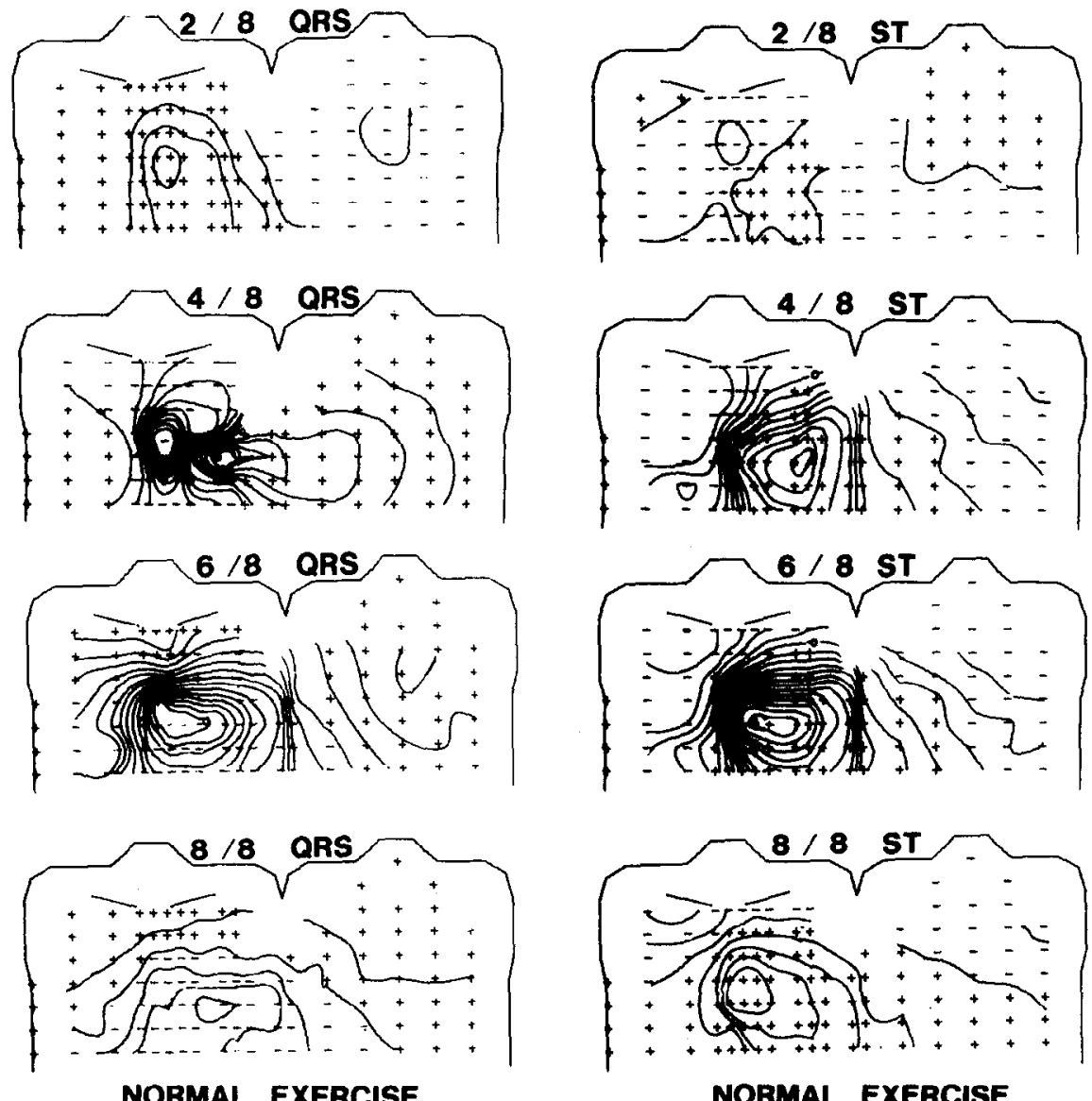

FICURE 2. Normal depolarization (ORS) pattern during peak exercise. The general pattern is similar to that at rest in the same patient in Figure 1. During repolarization (ST) a maximum develops in the left precordial region (2/8) that remains in the same location but broadens and increases in strength $(4 / 8,6 / 8)$ and diminishes at the end of repolarization (8/8). 
ysis of the transition of the body surface potential distribution from rest to peak exercise should be performed in order to determine whether abnormal patterns can be detected earlier from the surface maps than from the standard leads.

\section{Optimal Lead System for Exercise Electrocardlography}

The aforementioned data indicate that the sensitivity of exercise electrocardiography can be increased without loss of specificity when a large number of leads are analyzed simultaneously. The optimal lead system will then be based on a trade-off between a large number of leads and clinical applicability.

Patients with a normal electrocardiogram at rest: The selection of the lead system may be guided by the type of S-T patterns that may be expected in a given population. Exercise-induced myocardial ischemia in patients with a normal electrocardiogram at rest is characterized by a negative precordial potential during early repolarization (for example, S-T amplitude $60 \mathrm{~ms}$ after the end of the QRS complex). In such patients the single lead with the greatest sensitivity will be recorded between the right subclavicular area and the $V_{5}$ position (Fig. 4). This is the bipolar lead $\mathrm{CS}_{5}$ (Table I). However, in some patients the negative precordial potentials do not overlap with the $\mathrm{V}_{5}$ position and multiple precordial leads will be advantageous. This can be extended to a precordial map, such as the 16 lead map ( 4 by 4 ) used by Fox and co-workers. ${ }^{12,14}$

Patients with a previous myocardial infarction: In these patients the S-T changes may be caused by four

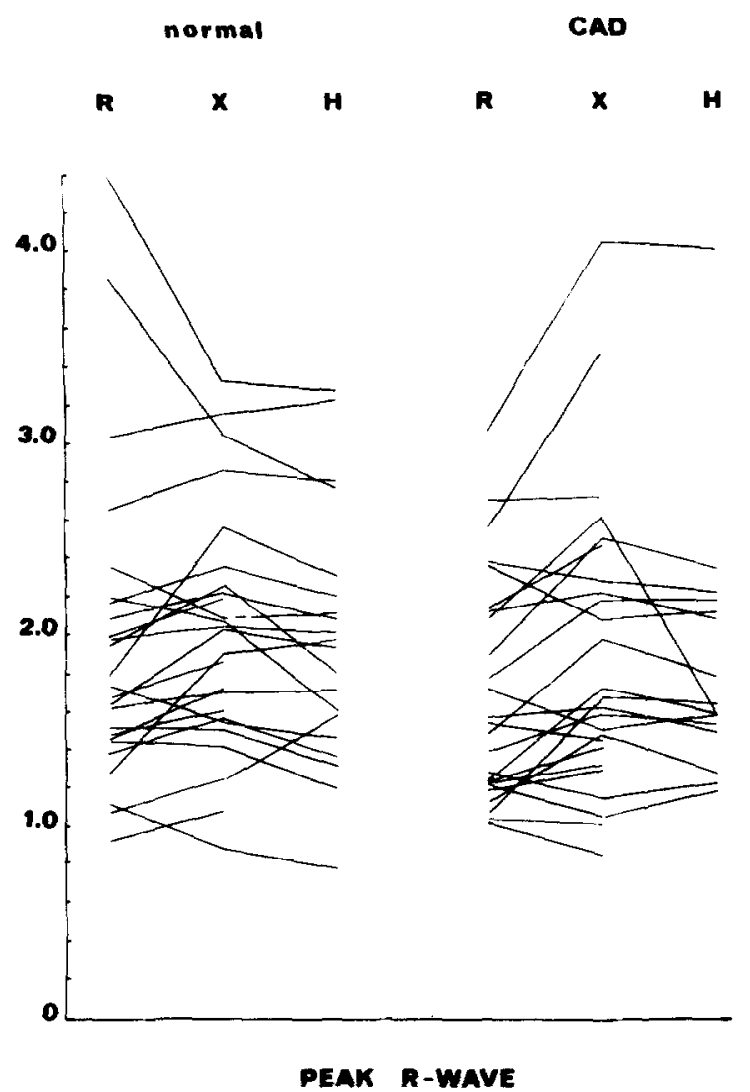

FIGURE 3. Maximal QRS amplitudes in the surface maps at rest (R), during peak exercise $(X)$ and in the 1st minute of recovery $(H)$ in normal subjects (left) and in patients with coronary artery disease (CAD) (right).
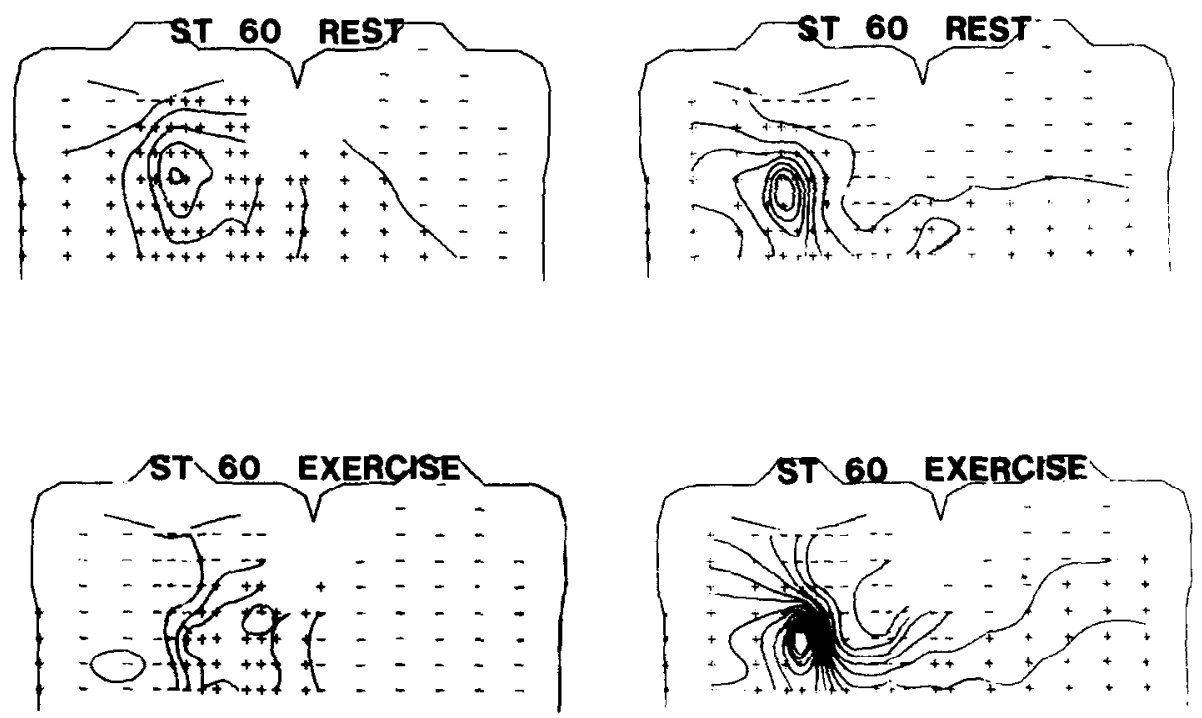

NORMAL

COR. ART. DISEASE

FICURE 4. Body surface maps $60 \mathrm{~ms}$ after the end of QRS complex (ST 60) at rest and at peak exercise in a normal subject (left) and in a patlent with coronary (COR.) artery (ART.) disease. Note a broad maximum in the left precordlal region in the normal subject, and negative precondial voltages in the patient. See legend to Figure 1. 

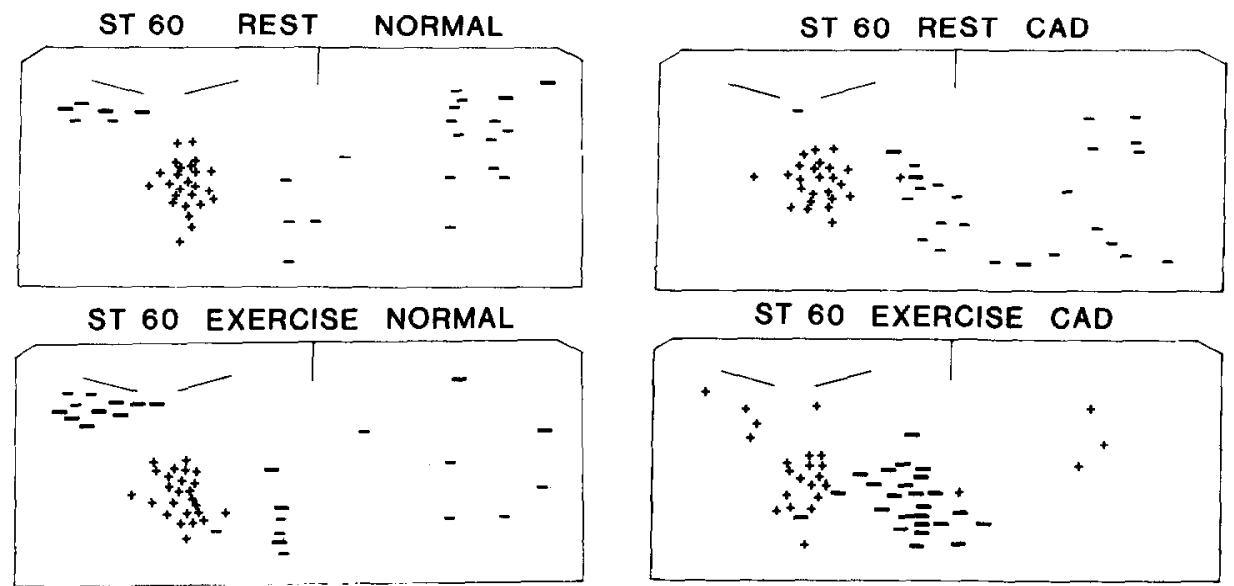

FICURE 5. Distribution of the maximums ( + ) and the minimums ( - ) of the body surface maps, $60 \mathrm{~ms}$ after the end of the QRS complex (ST 60). See legend to Figure 1. The minimums are indicated with a thick line for subjects with a minimal surface potential lower than - 0.050 mV, whereas a thin line was used for the other subjects. Note the maximums in the sternal area in the normal subjects (left) and in the patients (right) both at rest and during exercise. The minimums in the normal subjects are located at the back and in the right subclavian region. In the patients the minimums at rest are found at the back and the lower part of the left side of the chest. During exercise the minimums are deeper, and all are located in the precordial region.

mechanisms. (1) The factors that influence the S-T segment in normal subjects should be taken into account, such as heart rate, intracardiac blood volume and the position of the heart in the chest. (2) These are modified by the presence of scar tissue or hypokinetic or dyskinetic areas in the left ventricle without ischemia. This will largely cause S-T segment elevation in the leads that show the $Q$ waves of the previous infarction. ${ }^{15}$ (3) Myocardial ischemia may be provoked by exercise. In most patients, such ischemia occurs in the subendocardial layers of the entire left ventricle. This results in negative precordial potentials and S-T segment depression in the precordial leads. The location of the negative precordial potentials or the orientation of the abnormal S-T vectors appears to be independent of the location of the coronary arterial obstruction. $13,14,36$ However, the S-T vector orientation will be influenced by the presence of scar tissue due to previous infarction. ${ }^{15}$ Finally (4), in a few patients, transmural myocardial ischemia may develop as a result of coronary

\section{TABLE}

Comparison of Sensitivity and Specificity in Standard Precordlal Leads, in Lead $X$ of the Frank Orthogonal Lead System, in a Bipolar Chest Lead and in the Surface Map

\begin{tabular}{lcc}
\hline & $\begin{array}{c}\text { Speci- } \\
\text { ficity } \\
(n=25) \\
(\%)\end{array}$ & $\begin{array}{c}\text { Sensi- } \\
\text { tivity } \\
(n=25) \\
(\%)\end{array}$ \\
\hline $0.1 \mathrm{mV}$ mV horizontal or downsloping & 100 & 60 \\
S-T depression in leads $V_{2}$ to $V_{6}$ & 90 & 76 \\
S-T 60<-0.045 $\mathrm{mV}$ in lead $X$ & 95 & 84 \\
S-T 60 $<-0.045 \mathrm{mV}$ in lead CS & 88 & 100 \\
S-T 60<-0.050 mV in precordial map & 88 \\
S-T $60<0.090 \mathrm{mV}$ in precordial map & 100 & 84 \\
\hline
\end{tabular}

S-T 60 = S-T amplitude $60 \mathrm{~ms}$ after the end of the QRS complex. spasm. This will produce an area with S-T segment elevation over the ischemic zone. ${ }^{16}$

In patients with previous myocardial infarction, it might be advantageous to analyze the whole body surface potential distribution during exercise. A practical approach in research-oriented hospitals would be the reconstruction of the potential distribution from a set of 18 to 32 leads. ${ }^{17,18} \mathrm{~A}$ further reduction in the number

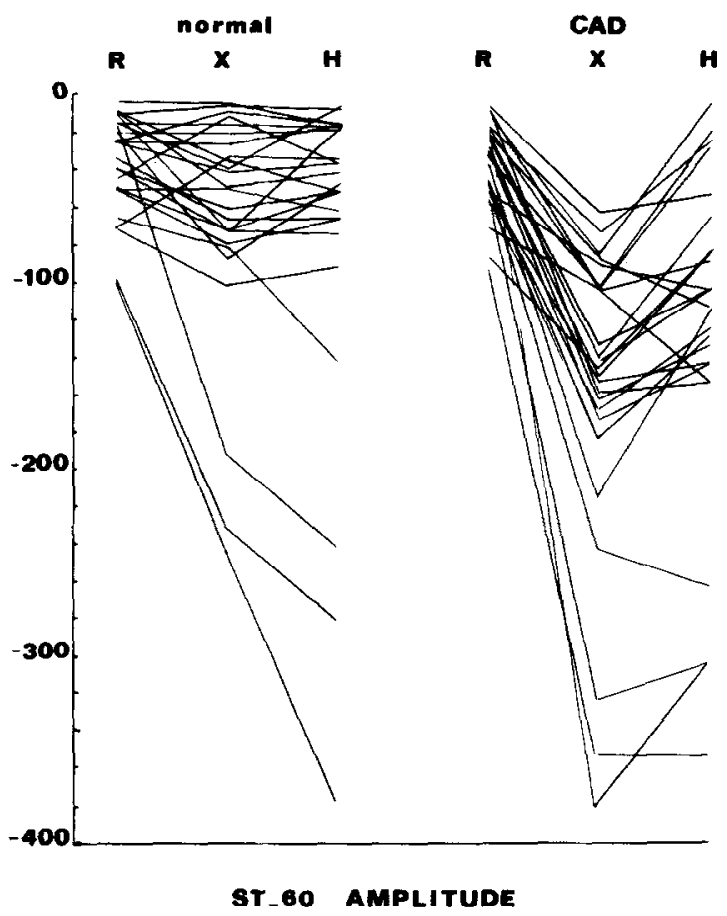

FIGURE 6. Amplitudes of the minimums of the surface maps at $60 \mathrm{~ms}$ after the end of the QRS complex in normal subjects (left) and in patients with coronary artery disease (right). See legend to Figure 3. 
of leads, with little loss of information, might be obtained through the method described by Kornreich ${ }^{19}$ for the resting electrocardiogram.

Because repolarization is largely characterized by a single maximum and a single minimum in the surface maps, the representation by a dipole will contain most of the information (Fig. 2 and 4). Thus, a corrected orthogonal lead system might be sufficient in all or most patients, provided that precise criteria are developed for spatial analysis of the S-T vectors. Such criteria have been described for patients with a normal electrocardiogram at rest ${ }^{20}$; however, data are lacking in patients with a previous myocardial infarction or other abnormalities in the electrocardiogram at rest. ${ }^{15}$

In summary, a single bipolar lead $\left(\mathrm{CM}_{5}\right.$ or $\left.\mathrm{CS}_{5}\right)$ will be sufficient in centers testing only subjects with a normal electrocardiogram at rest. In centers testing various types of patients, including those in the postmyocardial infarction state, such a system is not sufficient. In those laboratories one should record at least three (pseudo) orthogonal leads ${ }^{21,22}$ in addition to or including lead $\mathrm{CS}_{5}$, or a larger set of precordial leads. ${ }^{23,24}$ A corrected orthogonal lead system may be sufficient in the majority of patients, provided that specific criteria are developed that can be used in a computer system.

\section{Most Effective Electrocardiographic Measurements for Detection of Exercise-Induced Ischemia or Abnormal Myocardial Function}

The changes in the $P$ wave, QRS complex and ST-T segment during exercise in patients with coronary artery disease differ from the changes in normal subjects. ${ }^{15}$ In normal subjects the $\mathrm{P}$ wave amplitude typically increases, the maximal QRS amplitude decreases, the S wave deepens and the S-T junction depression occurs. ${ }^{25}$ However, the changes in individual subjects vary widely (Fig. 3).

Role of heart rate: The $P$ wave amplitudes generally increase less in patients with coronary artery disease, the QRS voltages may be enhanced and both S-T depression and S-T elevation may occur. For diagnostic use of these changes in the electrocardiogram, one should remember that patients with coronary artery disease generally stop exercise at a lower heart rate than do normal subjects. This factor alone has considerable influence on all electrocardiographic measurements. ${ }^{25}$ Therefore, the most effective measurements should be adjusted for differences in heart rate as shown in earlier studies. ${ }^{20}$

Changes in $P$ waves: Differences between the $P$ waves of patients and normal subjects during exercise have little if any diagnostic value (Table II). These differences are probably due largely to the lower peak heart rate in the patients, although they may be affected by elevated left ventricular filling pressure in some of the patients. ${ }^{26,27}$

Precordial QRS potentials: 'The surface map data indicate that the precordial QRS potentials increase during exercise in most patients as well as in normal
TABLE II

Sensitivity of P, QRS and S-T Measurements for Detection of Coronary Artery Disease In Men With a Normal Electrocardlogram at Rest $(n=53)$

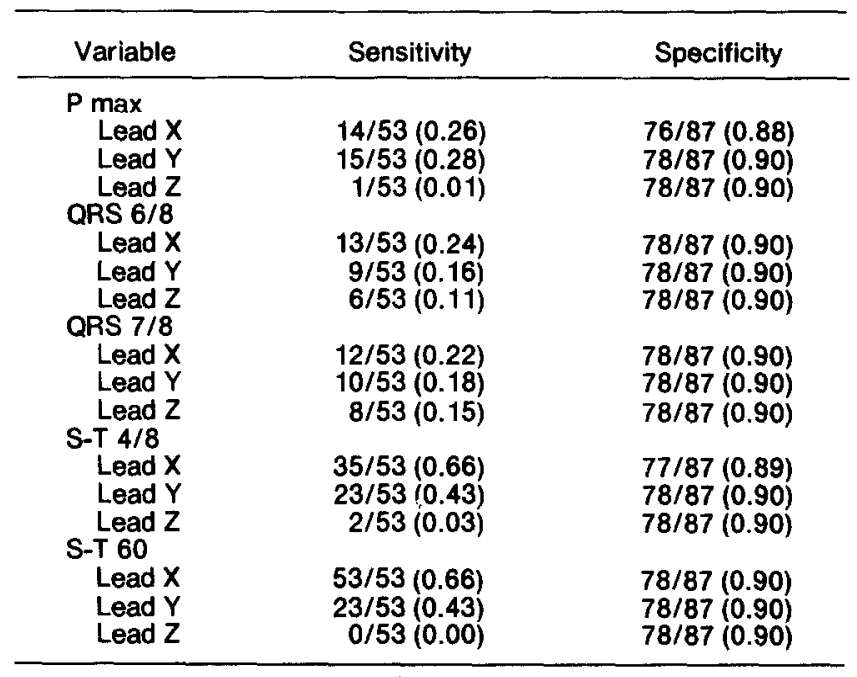

Measurements at the highest exercise level in each patient. Threshold values were selected so that the specificity was approximately 90 percent in 87 normal subjects. $P$ max $=$ point of maximal spatial magnitude of the $P$ wave; QRS 6/8 and QRS 7/8 = amplitude at $6 / 8$ and $7 / 8$, respectively, of QRS duration; S-T $4 / 8=$ amplitude at $4 / 8$ between the end of the QRS complex and the spatial maximum of the $T$ wave.

subjects. We found no systematic difference between the magnitude of these changes in the two groups (Fig. 3). This finding is at variance with recent reports that augmentation of the $R$ wave during exercise can be used as an indication for the presence of coronary artery disease. $^{28}$ The $\mathrm{R}$ wave changes in that study ${ }^{28}$ were analyzed in a single lead only. Thus, the observed differences in $R$ wave changes could be due to minor shifts in the location of the maximal QRS potentials at the chest wall (Fig. 1 and 2). Furthermore, the $R$ wave changes are related to heart rate. At submaximal work loads, $R$ wave amplitudes increase in normal subjects whereas decreased amplitudes occur at high heart rates. ${ }^{29}$ The differences between $R$ wave changes in normal subjects and in patients may therefore be due also in part to the lower peak heart rates in the latter group.

Finally, it has been suggested that the larger $R$ wave changes observed in coronary artery disease are caused by enlarged left ventricular volumes due to myocardial dysfunction. ${ }^{28}$ However, experimental data supporting this mechanism are lacking. One study ${ }^{30}$ reported no relation between $R$ wave changes and left ventricular volumes measured with blood pool scintigraphy. Data from our laboratory indicate that the $R$ wave changes like the $\mathrm{S}-\mathrm{T}$ changes, are dependent on the presence and location of a previous myocardial infarction (Table III). Thus more experimental and clinical data are required to define the significance of differences in QRS measurements among various groups of patients. ${ }^{31}$ 
Changes in S-T segment: A wealth of information is available on the $\mathrm{S}-\mathrm{T}$ segment changes during exercise. Both a horizontal or downsloping S-T segment depression and a slowly ascending $\mathrm{S}-\mathrm{T}$ segment have been associated with coronary artery disease. ${ }^{32}$ The likelihood of an abnormal (ischemic) S-T segment response is greater when the S-T segment depression (1) is greater in magnitude, measured at the J point or up to $80 \mathrm{~ms}$ after the QRS complex; (2) has a more horizontal or negative slope; (3) occurs at a reduced heart rate or earlier during the test; and (4) when it is associated with other symptoms such as chest pain and a lack of increase or decrease in systolic blood pressure.

Several studies have shown that the diagnostic value of exercise electrocardiography can be enhanced by computer processing, both in improving the signal/noise ratio and in applying specific measurements. Comparison of a large number of measurements has shown that the greatest diagnostic performance is obtained by a combination of S-T amplitudes and S-T slopes measured at fixed intervals after the QRS complex in the first $100 \mathrm{~ms}$ of the S-T segment. Such measurements were superior to S-T time integrals, time-normalized S-T amplitudes and polynomial fits of the S-T segment. ${ }^{20,33-36}$ The optimal points in which measurements should be taken are dependent on the algorithms for definition of the onset and end of the QRS complex. ${ }^{36,37}$ The data in Figure 7 indicate that the best single measurement was the S-T amplitude $60 \mathrm{~ms}$ after the end of the QRS complex, and that a further improvement could be obtained by a combination of S-T amplitude with heart rate and with S-T slope.

Through visual reading of the exercise electrocardiogram, changes in the S-T segment can be measured in steps of $0.1 \mathrm{mV}(1 \mathrm{~mm})$. Therefore, in clinical practice

\section{TABLE III}

\begin{tabular}{|c|c|c|c|}
\hline Variable & CAD & AMI & PMI \\
\hline $\begin{array}{l}P \text { max } \\
\text { Lead } X\end{array}$ & $19 / 53(0.35)$ & $19 / 49(0.38)$ & $26 / 61(0.42)$ \\
\hline $\begin{array}{l}\text { W/8 Lead X } \\
7 / 8 \text { Lead Y } \\
7 / 8 \text { Lead Z } \\
\text { S-T }\end{array}$ & $\begin{array}{r}19 / 53(0.35) \\
6 / 53(0.11) \\
18 / 53(0.33)\end{array}$ & $\begin{array}{r}28 / 49(0.57) \\
5 / 49(0.10) \\
4 / 49(0.08)\end{array}$ & $\begin{array}{l}22 / 61(0.16) \\
22 / 61(0.36) \\
23 / 61(0.37)\end{array}$ \\
\hline $\begin{array}{l}70 \text { Lead } X \\
90 \text { Lead } Y \\
70 \text { Lead } Z \\
\text { S-T }\end{array}$ & $\begin{array}{l}34 / 53(0.64) \\
37 / 53(0.69) \\
11 / 53(0.20)\end{array}$ & $\begin{array}{r}9 / 49(0.18) \\
11 / 49(0.22) \\
4 / 49(0.08)\end{array}$ & $\begin{array}{l}29 / 61(0.47) \\
28 / 61(0.45) \\
19 / 61(0.31)\end{array}$ \\
\hline $\begin{array}{l}\text { 4/8 Lead X } \\
5 / 8 \text { Lead Y } \\
5 / 8 \text { Lead Z }\end{array}$ & $\begin{array}{r}7 / 53(0.13) \\
23 / 53(0.43) \\
8 / 53(0.15)\end{array}$ & $\begin{array}{r}7 / 49(0.14) \\
3 / 49(0.06) \\
25 / 49(0.51)\end{array}$ & $\begin{array}{r}30 / 61(0.49) \\
23 / 61(0.37) \\
3 / 61(0.04)\end{array}$ \\
\hline
\end{tabular}

The variables with the highest sensitivity values in each group have been included. Threshold levels were selected so that the specificity was 90 percent in a group of 87 normal subjects. Unidentified abbreviations as in Table II. the same criteria are applied to various leads. When more accurate measurements are taken with the aid of a computer system, specific criteria should be developed for each lead or combination of leads. Such quantitative data provide the additional advantage of expressing the changes in the electrocardiogram as a probability figure indicating the degree of normality or abnormality. 35,38 This seems a more logical approach than a yes or no statement, based on arbitrary threshold values for S-T segment measurements.

\section{Discussion}

Approaches to optimal exercise electrocardiography: Two approaches to optimal exercise electrocardiography have been described. The first approach is the analysis of a large number of electrocardiographic leads. Whole body surface potential distributions during and after exercise have been studied in a small series of patients. Larger series have been reported by Fox et al., ${ }^{12,14}$ who recorded 16 precordial leads in a 4 by 4
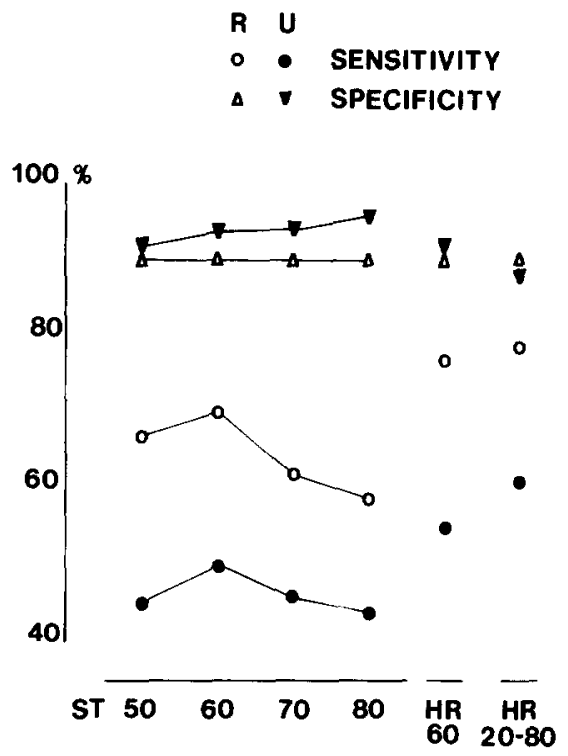

FICURE 7. Comparison of the sensitivity and specificity of various S-T amplitudes in two groups of subjects studied at the Thoraxcenter in Rotterdam (R) and at the St. Antonius Hospital in Utrecht (U), The Netherlands (see Ref. 36). In Rotterdam 73 patients with coronary artery disease were compared with 125 healthy volunteers without coronary arteriography. In Utrecht, the group consisted of 51 "normal" patients with chest pain but without stgnificant lesions at coronary arterlography, and of 96 patients with coronary artery disease. All subjects had a normal 12 lead electrocardiogram at rest. In addition, the Utrecht subjects had a normal exercise test at a previous occasion. This explains the lower sensitivity obtained in Utrecht for the same criteria. ${ }^{36}$ Visual reading of the electrocardiograms yielded sensitivity levels of 50 and 26 percent and specificity levels of 95 and 100 percent in the Rotterdam and Utrecht groups, respectively. Thresholds for the various computer measurements were selected so that the specificity was 90 percent in the 125 normal subjects from Rotterdam. Note that the best single measurement in both groups was the S-T amplitude $60 \mathrm{~ms}$ after the end of the QRS complex. A further improvement could be obtained by combination of the S-T amplitude with heart rate (HR 60) and by a combination of two S-T amplitudes, or S-T amplitude and slope, with heart rate (HR 20-80). 
array. A visual reading of these electrocardiograms yielded improved diagnostic value in comparison with the standard 12 leads. Such a lead system can easily be applied; however, it does not represent the whole body surface potential distribution. The second approach requires a more detailed computer analysis of a few leads. With such analysis, a similar diagnostic improvement has been reported as with the 16 lead precordial map.

The next step should be a combination of both approaches. This requires recording of precordial and whole body surface potential maps both during and after exercise in a large series of patients. The normal range of measurements from the QRS complex and the $\mathrm{S}$-T segment should be defined for each lead position. These normal ranges will probably be related to sex, age, body dimensions and heart rate during exercise and possibly to the type of test protocol that is used. From such material the optimal combination of leads and criteria can be defined.

It is possible that further studies will provide better criteria for exercise electrocardiography than those presented in this study. However, the additional gain will be limited because both precordial mapping and computer analysis already yield sensitivity levels of 80 to 90 percent at a specificity of 90 to 95 percent. ${ }^{12,20,35}$ This improvement in sensitivity of the exercise electrocardiogram by computer processing or by precordial mapping is of the same magnitude as the improvement obtained by nuclear imaging techniques. ${ }^{39-41}$

Clinical implications: Even the most sophisticated analysis of the exercise electrocardiogram cannot replace clinical judgment based on a combination of all data available in a given patient. These data should include a careful history, determination of exercise tolerance and the hemodynamic response to exercise as well as, in some patients, analysis of wall motion and global ventricular function from blood pool scintigrams $^{39}$ and an estimation of myocardial perfusion patterns and cell function from thallium scintigrams. $^{41}$

\section{Acknowledgment}

The data in Figure 7 were collected and analyzed as part of a cooperative study of the St. Antonius Hospital in Utrecht, the Thoraxcenter of the Erasmus University in Rotterdam and the Unit for Cardiovascular Research of the Free University in Brussels.

\section{References}

1. Elnthoven W. Weiteres über das Elektrokardiogram. Arch Ges Physiol Menschen Thiere 1908;122:517-20.

2. Schert D, Schaffer Al. The electrocardiographic exercise test. Am Heart J 1952;43:927-46.

3. Block P, Lenaers A, Tiberghlen J, et al. Surface maps and myocardial scanning at rest and during exercise: comparison with coronary angiography. Acta Cardiol 1976;31:467-81.

4. Tiberghien J, Steenhaut O, Lenders P, Block P, Kornrelch F. A 128-channel electrocardiographic recorder. Adv Cardiol (Karger) 1976;16:128-32.

5. Block P, Tlberghien J, Raadschelders I, et al. Diagnostic value of surface mapping recordings registered at rest and during exercise. In: Proceedings, Computers in Cardiology, 1978. Long Beach, CA: IEEE, 1978:89-93.

6. Lenaers A, Block P, van Thiel E, Lebedelle M, et al. Segmental analysis of T1-201 stress myocardial scintigraphy. J Nucl Med 1977; 18:509-16.

7. Taccardi B. Distribution of heart potentials in the thoracic surface of normal human subjects. Circ Res 1963;12:341-52.

8. Horan L, Flowers N, Brody O. Body surface potential distribution. Circ Res 1963;13:373-87.

9. Spach MS, Barr RC, Blumenschein SD, Bolneau JP. Clinical implications of isopotential surface maps. Ann Intern Med 1968; 69:919-28.

10. Miller $W T$, Spach MS, Warren RB. Total body surface potential mapping during exercise: QRS-T wave changes in normal young adults. Circulation 1980;62:632-45.

11. Mirvis DM, Keller FW Jr, Cox JW, Zettergen DG, Dowdie RF, Ideker RE. Left precordial isopotential mapping during supine exercise. Circulation 1977;56:245-52.

12. Fox KM, Solwyn A, Shillingford JP. Precordial electrographic mapping after exercise in the dlagnostic of coronary artery disease. Am J Cardiol 1979;43:541-6.

13. Simoone ML, Whthagen A, Vinke R, Kooy P, Bakker W. ST-Vector orientation and location of myocardial perfusion defects during exercise. Nukloarmedizin 1978;17:154-6.

14. Fox KM, Selwryn A, Oakley D, Shillinglord JP. Relation between the precordial projection of S-T segment changes after exercise and coronary angiographic findings. Am J Cardiol 1979;44:
1068-75.

15. Simoons ML, van den Brand M, Hugenholiz PG. Quantitative analysis of exercise electrocardiograms and left ventricular angiograms in patients with abnormal QRS complexes at rest. Circulation 1977;55:55-60.

16. Waters DD, Chaltman BR, Bourassa MG, Tubau JF. Clinical and angiographic correlates of exercise-induced ST-segment elevation. Circulation 1980;61:286-96.

17. Warren RB, Barr RC, Spach MS. Determining the minimum number and best placement of leads for a practical clinical body surface mapping system (abstr). Circulation 1977;56:Suppl III:III-200.

18. Yanowitz FG, Vincent GM, Lux RL, Merchant M, Davis D. Clinical applications of body surface ECG mapping during exercise testing. In: Proceedings of 1980 Engineering Foundation Conference "Computerized Interpretation of the ECG V." 1-8.

19. Kornreich $F$. The missing waveform information in the orthogonal electrocardiogram (Frank leads). I. Where and how can this missing waveform information be retrieved. Circulation 1973;48:98495.

20. Simoons ML. Optimal measurements for detection of coronary artery disease by exercise echocardiography. Comp Blomed Res 1977; 10:483-99.

21. Froellcher VF, Thompson AJ, Longo MR, Trlebwasser JH, Lancaster MC. Value of exercise testing for screening asymptomatic men for latent coronary artery disease. Prog Cardlovasc Dis $1976 ; 18: 265-76$.

22. Ellestad MH. Stress Testing: Principles and Practice. Philadelphia: FA Davis, 1975:67-9.

23. Tubau JF, Chaltman BR, Bourassa MG, Waters DD. Detection of multivessel coronary disease after myocardial infarction using exercise stress testing and multiple ECG lead systems. Circulation 1980;61:44-52.

24. Berman JL, Wynne J, Cohn PF. Multiple lead QRS changes with exercise testing. Circulation 1980;61:53-61.

25. Simoons ML, Hugenholtz PG. Gradual changes of ECG waveform during and after exercise in normal subjects. Circulation 1975; 52:570-7.

26. Roskamm H, Samek L, Rupp G, et al. Verbessert die zusätzliche Messung des Pulmonalkapillardruckes während körperlicher Be- 
lastung die Voraussage des koronarangiographischen Befundes bei Patienten ohne transmuralen Herzinfarkt? Z Kardiol 1977; 66:477-82.

27. Helkkliä J, Hugenholtz PG, Tabakin BS. Prediction of left heart filling pressure and its sequential change in acute myocardial infarction from the terminal force of the $\mathrm{P}$ wave. Br Heart $\mathrm{J} 1973$; 35:142-51.

28. Bonoris PE, Greenberg PS, Castellanet MJ, Ellestad MH. Significance of changes in R-wave amplitude during treadmill stress testing: angiographic correlation. Am J Cardiol 1978;41:84651.

29. Wolthuls AA, Froellcher VF, Hopkirk A, Flscher JR, Keiser N. Normal electrocardiographic waveform characteristics during treadmill exercise testing. Circulation 1979;60:1028-35.

30. Battler A, Froellcher V, Slutsky $\mathbf{R}$, Ashbum W. Relationship of QRS amplitude changes during exercise to left ventricular function and volumes and the diagnosis of coronary artery disease. Circulation 1979;60: 1004-13.

31. Simoons ML. QRS changes in CAD [Letter]. Circulation 1979; 59:841-3.

32. Rl|neke RD, Ascoop CA, Talmon JL. Clinical significance of upsloping ST segments in exercise electrocardiography. Circulation 1980;61:671-8.

33. Ascoop CA, Distelbrink CA, de Lang PA, Van Bemmel JH. Quantitative comparison of exercise vectorcardiograms and findings at selective coronary arteriography. $J$ Electrocardiol 1974:7:9-16.
34. Ascoop CA, Dletelbrink CA, de Lang PA. Clinical values of quantitative analysis of ST slope during exercise. Br Heart $\mathrm{J}$ 1977:39:212-7.

35. Hollenberg M, Budge WR, Wleneski JA, Certz EW. Treadmill score quantifies electrocardiographic response to exercise and improves test accuracy and reproducibility. Circulation 1980;61:276-85.

36. Simoons ML, Ascoop CA, Distelbrink CA, Block P, de Lang P, Vinke R. Computer processing of exercise electrocardiograms. In: Bemmel JM, Willems JL, eds. Trends in Computer Processed Electrocardiograms. Amsterdam: North Holland Publishing, 1977:383-406.

37. Simoons ML, Hugenholiz PG, Ascoop CA, Distelbrink CA, de Lang PA, Vinke R. Quantitation of exercise electrocardiography. Circulation, 1981;63:471-6.

38. Simoons ML, Hugenholtz PC. Estimation of the probability of exercise induced ischemia by quantitative ECG analysis. Circulation 1977;56:552-9.

39. Bodenheimer MM, Banka Vs, Helfant RH. Nuclear Cardiology. I. Angiographic assessment of left ventricular contraction: uses, limitations and future directions. Am J Cardiol 1980;45:661-73.

40. Bodenheimer MM, Banka VS, Helfant RH. Nuclear Cardiology. II. The role of myocardial perfusion imaging using thallium-201 in diagnosis of coronary heart disease. Am J Cardiol 1980;45: 674-84.

41. Okada RD, Boucher CA, Strauss HW, Pohost GM. Exercise radionuclide imaging approaches to coronary artery disease. Am J Cardiol 1980;46:1188-204. 